\title{
Research Paper: Comparing the Effect of Suspended and Non-Suspended Core Stability Exercises on Static and Dynamic Balance and Muscular Endurance in Young Males With Down Syndrome
}

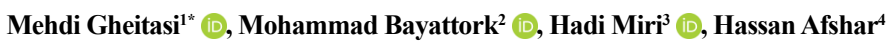 \\ 1. Department of Health and Sports Rehabilitation, Faculty of Sports Sciences and Health, Shahid Beheshti University, Tehran, Iran \\ 2. Department of Physical Education and Sports Sciences, Faculty of Humanities, University of Hormozgan, Bandar Abbas, Iran. \\ 3. Department of Physical Education and Sports Sciences, University of Amir Kabir, Tehran, Iran. \\ 4. Department of Corrective Exercise and Sports Injuries, Faculty of Physical Education and Sports Sciences, Islamic Azad University of Karaj, Alborz, Iran.
}

\begin{tabular}{|c|c|}
\hline $\begin{array}{l}\text { Use your device to scan } \\
\text { and read the article online }\end{array}$ & Citation Gheitasi M, Bayattork M, Miri H, Afshar H. Comparing the Effect of Suspended and Non-Suspended Core Stability \\
\hline 口ita & Exercises on Static and Dynamic Balance and Muscular Endurance in Young Males With Down Syndrome. Physical Treat- \\
\hline atifin & doi : $:$ http://dx.doi.org/10.32598/ptj.9.3.153 \\
\hline
\end{tabular}

\section{(1) (\$)}

Article info:

Received: 15 Feb 2019

Accepted: 07 May 2019

Available Online: 01 Jul 2019
Keywords:

Down syndrome, Core stability exercises, Balance, Muscular endurance

\begin{abstract}
A B S T R A C T
Purpose: People who have Down Syndrome (DS) usually have deficiencies in muscular strength, muscular endurance, the development of motor skills, postural control, and gait cycle. These muscular and balance deficits impair daily living activities in people with DS; therefore, attention to improving these factors in various recreational environments seems necessary. Thus, the present study aimed to compare two methods of suspended and non-suspended core stability exercises (Total Resistance exercises (TRX) and Swiss ball) on balance and muscular endurance in males with DS.
\end{abstract}

Methods: This was a quasi-experimental research with a pretest-posttest and a control group design. The statistical sample of the study included 54 males aged 18-27 years with DS. Moreover, the samples were purposefully selected based on the study inclusion and exclusion criteria and were randomly divided into three groups (each group: 18 individuals). Stork test, Y Balance Test (YBT), and core stabilizer muscle endurance tests were used to evaluate static balance, dynamic balance, and muscular endurance, respectively. After measuring the pretest, the first and second groups and the control group continued core stability exercises with Swiss ball, core stability exercises with TRX, and their daily routine life activities for 8 weeks, respectively. Eventually, the posttest was performed after the completion of the trial.

Results: The obtained results suggested that the core stability training groups with Swiss ball and TRX had a significant increase in the posttest scores of static balance, dynamic balance, and muscular endurance, compared to the control group. Post-hoc test results also illustrated that the mentioned difference was between both training groups and the training groups with the control group; therefore, in the variable of static balance, the core stability training group with Swiss ball demonstrated a more considerable increase than the core stability training group with TRX. However, in the variables of dynamic balance and muscular endurance, the core stability training group with TRX revealed more increase.

Conclusion: This study indicated that the core stability exercises with Swiss ball improved static balance, dynamic balance, and muscular endurance in males with DS. Thus, such exercises can be used in a different enjoying environment to develop some fitness indicators in this group.

\section{* Corresponding Author:}

Mehdi Gheitasi, PhD.

Address: Department of Health and Sports Rehabilitation, Faculty of Sports Sciences and Health, Shahid Beheshti University, Tehran, Iran

Phone: +98 (912) 3547613

E-mail:m_gheitasi@sbu.ac.ir 


\section{Highlights}

- Core stability training with a Swiss ball and TRX can improve the static balance, dynamic balance, and muscular endurance abilities in males with Down syndrome.

- Core stability training with Swiss ball indicated a more considerable increase in static balance ability, compared to the core stability training with TRX.

- Core stability training with TRX suggested a more considerable increase in dynamic balance and muscular endurance abilities, compared to the core stability training with the Swiss ball.

\section{Plain Language Summary}

People with Down Syndrome can benefit from core stability training protocols using a Swiss ball and TRX to improve their physical abilities, especially balance and muscular endurance abilities. Accordingly, they could improve their quality of life.

\section{Introduction}

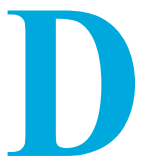

own Syndrome (DS) is among the most prevalent developmental disorders. It usually (90-95\% of the cases) occurs due to the presence of an extra chromosome on chromosome 21; such condition is called trisomy 21 [1]. The primary cause of DS is unrecognized. The old age of the mother in the time of fertility, the effects of X-ray on the egg cell and germ cells during the first months of pregnancy, hormonal disorders, and viral infections are among the reasons for the development of this disorder [2]. These children have similar anatomical and muscular characteristics.

Moreover, the development of motor skills in children with DS is similar to the growing pattern of healthy children; however, it is slow and delayed [3]. Moreover, children with DS often indicate weakness in muscular strength, muscular endurance, the development of motor skills, disorders in controlling posture, and gait cycle [4]. Balance problems and postural stability prevent people with DS from exercises and movements in their daily living; therefore, it is necessary to provide movement programs in a happy and enjoyable environment to enhance their willingness to participate in such programs and improving their motor skills.

Exercise interventions are capable of improving physical skills, promoting socialization, and providing health benefits. Furthermore, they overall provide a pathway, which improves the quality of life of people with DS [5, 6]. In addition, a review study demonstrated the necessity of using specific exercise interventions to improve the physical abilities of people with DS [7]. Practicing core stabilization has been considered to improve the balance performance of people with disabilities [8]. The core muscles provide the stability of the trunk and spine during movements, such as jumping, running, and throwing. Weakness in muscular endurance, especially in the trunk area, can affect the stability of this area while performing motor skills. Ultimately, it impairs one's function [9].

Additionally, core stability exercises positively impact pain relief, abdominal muscle activation, lumbar spine stability, and performance [10]. Furthermore, the stability of the core area can help to improve dynamic balance, coordinate the upper and lower trunk muscles, and reduce muscles' damage and abnormalities [9]. Gupta et al. examined the impact of an exercise intervention on the strength and balance of people with DS [11]. Rahman et al. investigated the effect of a 6-week weight training on the balance of people with DS. They reported a significant increase in the static and dynamic balance in the exercise group [12]. In addition, the results of other surveys examining the role of exercise programs in the balance of children with DS revealed that moving programs improved their balance $[8,13]$.

Moreover, applying new exercise approaches such as Total Resistance exercises (TRX) has drawn the attention of health and fitness researchers. TRX is a new technique in which a rope is used for contraction and movement [14]. In comparison to conventional resistance training, performing TRX exercises enables a person to move at higher angels. Furthermore, the risk of injury in resistance training with TRX is less, which is because of muscle stimulation through body weight [14]. Therefore, 
the general public and professional athletes are practicing TRX to improve their muscular endurance, balance, and postural control. Therefore, improving balance and muscular endurance in daily activities and participating in exercise programs is beneficial for people with DS.

Moreover, improving their capability to control and acquire motor skills during their critical age in which they are trying to perform all their tasks by themselves is of importance. They need to strengthen and improve these movement indicators $[2,7]$. Sports tools have been flourishing daily, and newer and more different approaches have been introduced to the world, such as Swiss ball and TRX. Such approaches help with fitness and are portable, fun and straightforward. Using a modified type of these methods could influence the improvement of balance weaknesses and muscular endurance in people with DS. Despite the proven benefits of exercise training in people with DS, their presence in the stable environments of conventional resistance training is challenging. Thus, it makes some of them unwilling to participate in such activities [15].

Providing different training and recreational conditions, like TRX and Swiss ball training in a modified environment could lead to greater motivation for them to participate in sports and ultimately improve some of their fitness factors. No survey has compared two different training methods with routine practices, like suspension exercises with TRX and non-suspension exercises with Swiss ball on the balance performances and muscular endurance of people with DS. Therefore, the current study compared two methods of core suspension stability training with TRX and non-suspension one with Swiss ball upon static and dynamic balance and muscular endurance in males with DS.

\section{Materials and Methods}

The present quasi-experimental study was conducted in a professional rehabilitation center in Qazvin City, Iran, in 2018. The research design comprised three groups, including two experimental groups of TRX and Swiss ball and a control group without a training intervention. Moreover, Pre-test and Post-test were conducted on all groups. The study was approved by the Ethics Committee of Islamic Azad University, Karaj branch. The study participants were 54 males with DS, aged 18-24 years. Their Intelligence Quotient (IQ) ranged from 50-70 based on the Wechsler Intelligence Scale [16].

Moreover, they were selected by convenience purposive sampling method. The sample size was calculated equal to 15 people in each group according to the primary variable of research using $\mathrm{G}^{*}$ power software with the test power of $90 \%$ and confidence interval of $95 \%$; however, this size increased to $20 \%$ (equal to 18 individuals per group), because of considering dropout. In total, 54 people completed the experimental procedure. The inclusion criteria included no moderate or severe mental retardation (IQ: <50), no muscular problems, like muscle atrophy, no deformities, and abnormalities affecting research process, no participation in training exercises in the past 6 months, no history of trunk and lower limb injuries in the past year, no pain, no history of surgery in the trunk and lower limb, and having healthy vision and hearing with or without assistive devices $[8,17]$.

The exclusion criteria were not completing the consent form, unwillingness to continue study at any point in the research process, and is absent in more than three sessions of the training program. Initially, the study participants filled out a demographic and personal information form. To match the individuals, their height and weight were measured using a measuring tape and a digital scale. Moreover, to select the dominant leg, the hit the ball test was performed. Figure one shows the process of research implementation.

After selecting the samples, the Pre-test was performed. Then, the study subjects were randomly divided into three groups, including TRX modified training, Swiss ball modified training, and controls. Stork Balance Test was used to assess static balance, in which the subject stands with one foot on a flat surface and raises his/her free leg to the ankle, and his/her hands are free on both sides of body. The tester measures the maximum time that the subject stands on feet, and stops when the subject places the free leg on the ground. This experiment is repeated three times, and the best time is set as a record for them. The validity of the Stork test was reported to be $87 \%$ in several tests $[18,19]$.

To evaluate dynamic balance, the Y Balance Test was used in which three directions (anterior, posteromedial, \& posterolateral) were used to measure the subjects' balance. The graded bars illustrate the angels of these three directions, which are fixed in the lateral sections of the plate in three directions; a marker is installed on each bar. Before the test, the study subjects' dominant leg was determined; if it was the right foot, the experiment should have been performed anticlockwise, while if it was the left one, the experiment should have been performed clockwise. The subjects stood on their upper leg on the three-way crossing plate, and until no mistake was made (his foot did not move from the three-way crossing 


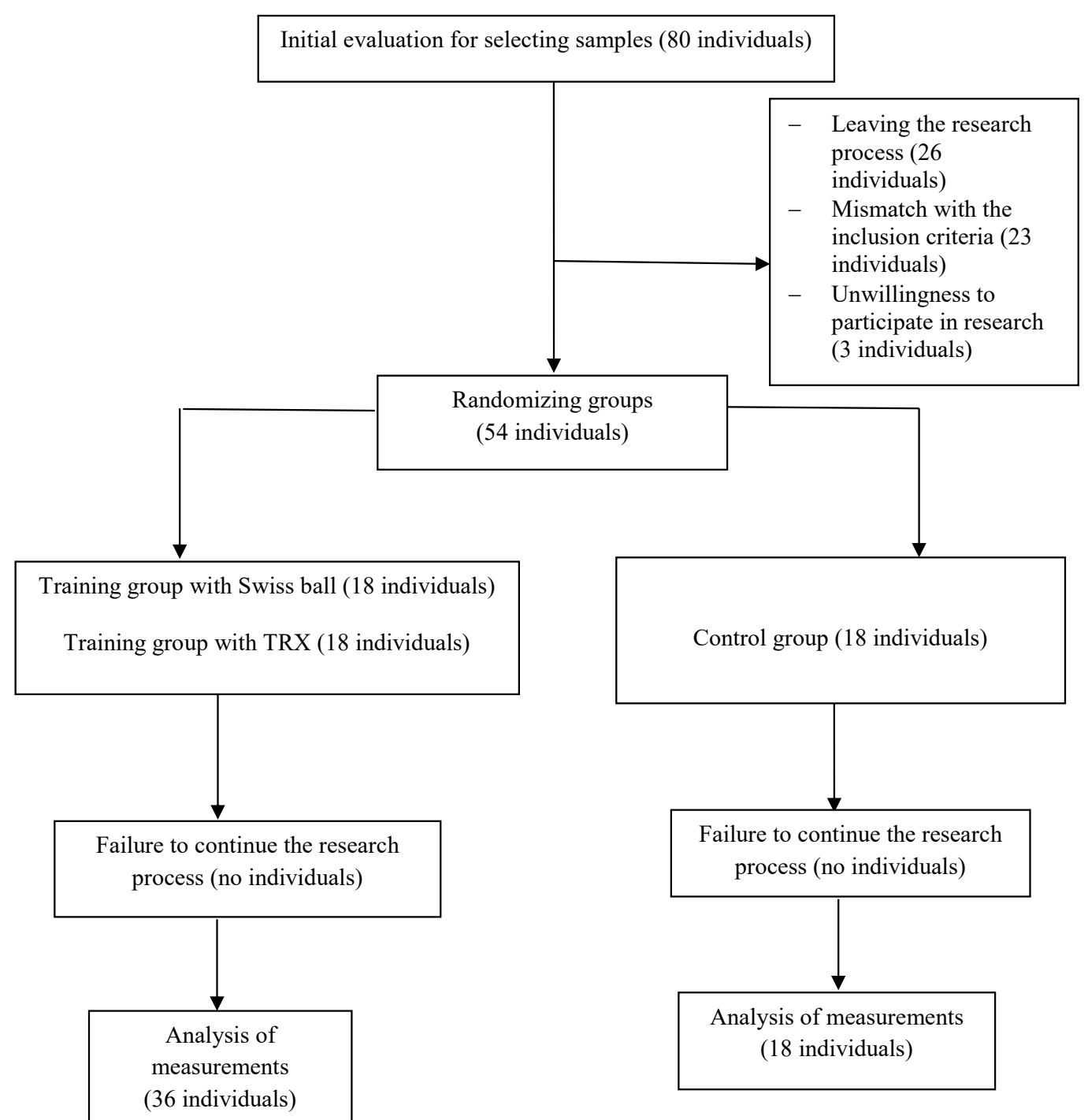

Figure 1. The diagram of the research process

PHYSICAL TREA MENTS

plate, did not rely upon the leg, which made the access or did not fall), they performed the accessing task with their other leg. This task was performed in the direction that the tester randomly determined to employ markers' movement. Then, they returned to the normal position of standing on two legs. The distances in which the subject moved the marker was recorded as his balance score. Each subject practiced every direction exercise for three times. After the relevant mean value was calculated in centimeters, it was multiplied in 100 and was considered as a percentage of the foot length.

Modified Biering Sorensen test was used to measure the endurance of the posterior trunk. McGill et al. documented its reliability equal to $93 \%$ [15]. Anterior trunk flexor test with a reliability of 0.98 (reported by McGill et al., 1999) was used to measure the endurance of an- terior stabilizer muscles of the trunk. The Side Bridge Test (left and right) was used to measure the endurance of the lateral stabilizer muscles of the trunk. In addition, its reliability has been reported as $95 \%$ by McGill and associates. In performing all of the tests, time was recorded. The total endurance of stabilizer muscles of the trunk in all dimensions (posterior, anterior, \& lateral) was used as a single unit [14].

The training group subjects conducted exercises under the supervision of a corrective exercise expert and a physiotherapist for 8 weeks and three times per week at the rehabilitation center. Adequate explanations for performing training programs were provided to the individuals to ensure that they were able to understand and execute the training programs correctly. Furthermore, due to the nature of studies with exercise intervention, 
the study participants could not be blinded. The exercise protocol included a 5-min warm-up using some games and recreational practices to provide a happy environment and increasing the motivation for the next exercises. The training protocol for the Swiss ball group was selected based on Jeffrey's suggested training program. This modified protocol consists of three levels, starting with level one practice, and gradually progressing to level three exercises. Exercises of level one involve static contractions in a stable position. Level two exercises consist of stable movements in a dynamic environment. Level three exercises comprise dynamic movements in an unstable environment and resistance movements in this environment (sit-ups on Swiss ball, back-extension on Swiss ball, and raising the opposing hand and leg on Swiss ball) $[8,20]$.

The training protocol for the TRX group comprised 4 practices, in the following order:

1. Put heel into the TRX grips, lift the pelvis the ground, and hold this position for 10 seconds.

2. Put the ankle into the TRX bands and bend to one side. In this position, the head, trunk, lower limb, and foot are aligned in the same direction for 10 seconds.

3. Repeat exercise number 2 for the other side of body.

4. Put the ankles into the TRX bands; then, hold a prone position in which the head, trunk, thigh, knee, and ankle are aligned in the same direction (plank exercise).

The control group performed their daily activities during this period. Finally, the Post-test was performed.

In addition, descriptive statics were used to determine central indices. The Kolmogorov-Smirnov test was applied to check the normality of the obtained data. Considering the normality of the data and the assumption of the homogeneity of variances, Analysis of Covariance (ANCOVA) was used to compare the groups' Post-test mean scores. All statistical analyses were performed by SPSS at the significance level of 0.05 .

\section{Results}

The study subjects' characteristics (height, weight, \& age) along with their homogeneity assessment are demonstrated in Table 1.

The Pre-test-Post-test comparison results, including static balance, dynamic balance, and muscle endurance in two exercise and control groups, are illustrated in Table 2.

The obtained results suggested that the Swiss ball and TRX training groups had a significant increase in Posttest scores of all components, comprising static balance, dynamic balance, and muscle endurance, compared to the controls. Moreover, the effect size of this study was considered as a significant effect (in the range of 0.43 0.66 ; based on the available range of this index, the effect size of $\eta 2 \geq 0.06$ is defined as the small effect size, $0.14 \geq \eta 2 \geq 0.06$ is defined as the medium one, and 0.14 $\geq \eta 2$ is defined as large effect size) [21]. The post-doc test results for identifying the location of differences indicated differences between the mean values of the training group, and Swiss ball and control groups $(\mathrm{P}=0.0000)$, as well as between the mean scores of the training group, and TRX and control groups $(\mathrm{P}=0.000)$. Furthermore, a significant statistical difference was observed between two training groups $(\mathrm{P}=0.0000)$. A more considerable increase was achieved in the static balance variable in the Swiss ball training group. Moreover, the increase was higher in the dynamic balance and muscle endurance in the TRX training group.

\section{Discussion}

The present study investigated the impact of core stabilization exercises with Swiss ball and TRX on the balance and muscle endurance abilities of people with DS. The collected results revealed a significant improvement

Table 1. Demographic characteristics of the study subjects and their homogeneity assessment (Mean \pm SD)

\begin{tabular}{|c|c|c|c|c|c|}
\hline \multirow{2}{*}{ Subject's Characteristics } & \multicolumn{3}{|c|}{$(n=18)$} & \multirow{2}{*}{ Test Statistics (F) } & \multirow{2}{*}{$\mathbf{P}$} \\
\hline & Swiss Ball Exercises & TRX Exercises & Control Group & & \\
\hline Height $(\mathrm{cm})$ & $148.8 \pm 1.11$ & $153.1 \pm 5.8$ & $160 \pm 7.4$ & 2.98 & 0.91 \\
\hline Weight (kg) & $63.3 \pm 5.57$ & $66 \pm 5.35$ & $67.1 \pm 5.97$ & 1.93 & 0.138 \\
\hline Age (year) & $20.5 .8 \pm 1.17$ & $22.6 \pm 1.5$ & $23.2 \pm 2.07$ & 1.23 & 0.306 \\
\hline
\end{tabular}


Table 2. The ANCOVA results for comparing static balance, dynamic balance, and muscle endurance in three groups

\begin{tabular}{|c|c|c|c|c|c|c|}
\hline \multirow{2}{*}{ Variable } & \multirow{2}{*}{ Group } & \multicolumn{2}{|c|}{ Mean \pm SD } & \multirow{2}{*}{$\mathbf{F}$} & \multirow{2}{*}{$\mathbf{P}$} & \multirow{2}{*}{ Effect Size } \\
\hline & & Pre-test & Post-test & & & \\
\hline \multirow{3}{*}{ Static balance } & Swiss ball exercises & $5.93 \pm 1.15$ & $9.11 \pm 1.21$ & & & \\
\hline & TRX exercises & $5.60 \pm 1.29$ & $7.75 \pm 1.66$ & 50.584 & 0.000 & 0.66 \\
\hline & Control & $5.50 \pm 0.99$ & $5.34 \pm 0.93$ & & & \\
\hline \multirow{3}{*}{ Dynamic balance } & Swiss ball exercises & $50.54 \pm 4.29$ & $54.39 \pm 3.45$ & & & \\
\hline & TRX exercises & $52.79 \pm 4.19$ & $58.43 \pm 4.18$ & 19.374 & 0.000 & 0.43 \\
\hline & Control & $51.82 \pm 4.21$ & $50.95 \pm 4.45$ & & & \\
\hline \multirow{3}{*}{$\begin{array}{l}\text { Muscular } \\
\text { endurance }\end{array}$} & Swiss ball exercises & $19.52 \pm 1.36$ & $23.02 \pm 1.91$ & & & \\
\hline & TRX exercises & $19.46 \pm 1.24$ & $26.73 \pm 2.54$ & 49.924 & 0.000 & 0.66 \\
\hline & Control & $21.01 \pm 2.18$ & $20.62 \pm 2.09$ & & & \\
\hline
\end{tabular}

in the ability of static balance, dynamic balance, and muscular endurance in the study subjects after attending an 8-week training program with a Swiss ball and TRX, in comparison with the controls. These results are in line with the previous studies that have investigated the effectiveness of various exercise methods, like balance exercises and postural stability, upon improving balance and muscular endurance in people with mental disabilities, including DS [9, 11, 22].

Improvements in balance function and muscular endurance in people with DS may be due to complex sensorymotor changes after trying for adaptation and balance maintenance in unbalanced conditions like being placed on the Swiss ball or TRX. This is consistent with the achievements of a study suggesting that exercises in unbalancing conditions could significantly enhance the sense of depth in individuals [23]. Previous researchers have argued that people with mental disabilities are unable to detect different mechanical requirements in unstable situations, indicating a deficiency in their proprioception system. Such problem could affect the ability to acquire sufficient information, responding to visual stimuli and proprioception, and ultimately leading to reduced output balance and proper postural maintenance $[24,25]$. Therefore, the improved balance functions of people with DS in the present study could be due to the intense stimulation of the proprioception during exercising with Swiss ball and TRX.

Additionally, core stabilization exercises not only increase endurance nerve control in this area but also affect the body's ability to maintain balance by controlling the spine and trunk [26]. Furthermore, high importance of the function of the central part of the body for maintaining the stability and producing power in all activities has been proved [27]. Physiologically, core stability increases the strength, endurance, and efficient use of upper and lower limbs' muscles. Therefore, biomechanically, increasing the muscular endurance of the central part of the body, and subsequently improving balance performance can be considered as a principle in all daily activities and exercises for people with mental disabilities [28].

Another obtained result was that more improvement occurred in the static balance in the Swiss ball training group as well as muscular endurance and dynamic balance in the TRX training group. Such variation appears to be due to the nature of the exercises by the Swiss ball and with TRX ropes [29]. Although using both exercises is naturally unstable, exercising with TRX has more instability. Previous research studies have reported that increasing instability in exercises leads to more neuromuscular involvement in target muscles; therefore, it can enhance functional abilities, like dynamic balance or increase muscular endurance $[29,30]$. Furthermore, in addition to neuromuscular adaptations, an essential factor in improving dynamic balance more than static balance has higher muscular strength, particularly in the lower limbs. Thus, training with TRX could provide more improvements in the dynamic balance because of its unstable nature $[31,32]$. 
Overall, the training protocols used in the present study were different from the previous investigations; because they were based on the performance of modified central stabilization exercises on the Swiss ball and TRX. However, it seemed that most protocols included conventional resistance approaches, which were accepted by people with DS. Accordingly, all study participants expressed their interest in participating in the program. Only the investigations by Kubilay et al. [9] and Giagazoglou et al. [15] were to some extent, similar to this study in terms of using Swiss ball and Trampoline.

One of the main positive points of this study was that for the first time, recreationally modified exercises were used for people with DS. A limitation of this study was the use of field tests to evaluate research variables. It is suggested that further studies apply more accurate and reliable tools, like the Biodex system or force plate. Moreover, the present study only explored males with DS. It is suggested to conduct similar studies on females with DS. Moreover, the effectiveness of the proposed protocols in this study on other health and fitness or even mental factors in people with DS can be examined by other researchers. Furthermore, the study results only included people with mild DS, and future research studies can examine the effectiveness of training with Swiss ball and TRX upon physical fitness of people with moderate or severe DS or those with other mental disorders by adhering to all the health and safety principles.

By improving balance and muscular endurance abilities, people with DS can benefit from core stability training protocols using a Swiss ball and TRX to enhance their physical abilities and, ultimately, their quality of life.

\section{Ethical Considerations}

\section{Compliance with ethical guidelines}

All ethical principles were considered in this article, and all of the study participants provided the written consent form. Also, this study was confirmed by the Ethics Committee of Islamic Azad University of Karaj.

\section{Funding}

This research received no specific grant from funding agencies in the public, commercial, or not-for-profit sectors.

\section{Authors' contributions}

All authors contributed to designing, running, and writing all parts of the research.

\section{Conflict of interest}

The authors declared no conflict of interest.

\section{Acknowledgments}

The authors would like to thank the staff and authorities of the rehabilitation Center in Qazvin, Iran, and all study participants for their valuable cooperation.

\section{References}

[1] Parker SE, Mai CT, Canfield MA, Rickard R, Wang Y, Meyer $\mathrm{RE}$, et al. Updated national birth prevalence estimates for selected birth defects in the United States, 2004-2006. Birth Defects Research Part A. 2010; 88(12):1008-16. [DOI:10.1002/ bdra.20735] [PMID]

[2] González-Agüero A, Vicente-Rodríguez G, Moreno L, Guerra-Balic M, Ara I, Casajus J. Health-related physical fitness in children and adolescents with Down syndrome and response to training. Scandinavian Journal of Medicine \& Science in Sports. 2010; 20(5):716-24. [DOI:10.1111/j.16000838.2010.01120.x] [PMID]

[3] Sugimoto D, Bowen SL, Meehan WP, Stracciolini A. Effects of neuromuscular training on children and young adults with Down syndrome: systematic review and meta-analysis. Research in Developmental Disabilities. 2016; 55:197-206. [DOI:10.1016/j.ridd.2016.04.003] [PMID]

[4] Albin CM. The benefit of movement: Dance/movement therapy and Down syndrome. Journal of Dance Education. 2016; 16(2):58-61. [DOI:10.1080/15290824.2015.1061196]

[5] Li C, Chen S, How YM, Zhang AL. Benefits of physical exercise intervention on fitness of individuals with Down syndrome: A systematic review of randomized-controlled trials. International Journal of Rehabilitation Research. 2013 36(3):187-95. [DOI:10.1097/MRR.0b013e3283634e9c] [PMID]

[6] Hardee J, Fetters L. The effect of exercise intervention on daily life activities and social participation in individuals with Down syndrome: A systematic review. Research in Developmental Disabilities. 2017; 62:81-103. [DOI:10.1016/j. ridd.2017.01.011] [PMID]

[7] Pitetti K, Baynard T, Agiovlasitis S. Children and adolescents with Down syndrome, physical fitness and physical activity. Journal of Sport and Health Science. 2013; 2(1):47-57. [DOI:10.1016/j.jshs.2012.10.004]

[8] Aly SM, Abonour AA. Effect of core stability exercise on postural stability in children with Down syndrome. International Journal of Medical Research and Health Sciences. 2016; 5:213-22.

[9] Kubilay NS, Yildirim Y, Kara B, Harutoglu Akdur H. Effect of balance training and posture exercises on functional level in mental retardation. Fizyoterapi Rehabilitasyon. 2011; 22(2):55-64. 
[10] Bliss LS, Teeple P. Core stability: The centerpiece of any training program. Current Sports Medicine Reports. 2005; 4(3):179-83. [DOI:10.1097/01.CSMR.0000306203.26444.4e]

[11] Gupta S, Rao Bk, Kumaran S. Effect of strength and balance training in children with Down's syndrome: A randomized controlled trial. Clinical Rrehabilitation. 2011; 25(5):425-32. [DOI:10.1177/0269215510382929] [PMID]

[12] Rahman SAA, Shaheen AA. Efficacy of weight bearing exercises on balance in children with Down syndrome. Egyptian Journal of Neurology, Psychiatry and Neurosurgery. 2010; 47(1):37-42.

[13] Wang WY, Ju YH. Promoting balance and jumping skills in children with Down syndrome. Perceptual and Motor skills. 2002; 94(2):443-8. [DOI:10.2466/pms.2002.94.2.443] [PMID]

[14] McGill SM, Cannon J, Andersen JT. Analysis of pushing exercises: muscle activity and spine load while contrasting techniques on stable surfaces with a labile suspension strap training system. Journal of Strength and Conditioning Research. 2014; 28(1):105-16. [DOI:10.1519/ JSC.0b013e3182a99459] [PMID]

[15] Giagazoglou P, Kokaridas D, Sidiropoulou M, Patsiaouras A, Karra C, Neofotistou K. Effects of a trampoline exercise intervention on motor performance and balance ability of children with intellectual disabilities. Research in Developmental Disabilities. 2013; 34(9):2701-7. [DOI:10.1016/j. ridd.2013.05.034] [PMID]

[16] Savucu Y. Influence of 12-week training on aerobic capacity and respiratory functions of adolescents with Down syndrome. World Appl Sci J. 2010; 11(10):1292-6. [DOI:10.1590/ S0103-05822014000100015]

[17] Määttä T, Tervo-Määttä T, Taanila A, Kaski M, Iivanainen M. Mental health, behaviour and intellectual abilities of people with Down syndrome. Down Syndrome Research and Practice. 2006; 11(1):37-43. [DOI:10.3104/reports.313] [PMID]

[18] Villamonte R, Vehrs PR, Feland JB, Johnson AW, Seeley MK, Eggett D. Reliability of 16 balance tests in individuals with Down syndrome. Perceptual and Motor Skills. 2010; 111(2):530-42. [DOI:10.2466/03.10.15.25.PMS.111.5.530-542] [PMID]

[19] Boer P-H, Moss SJ. Validity of the 16-metre PACER and sixminute walk test in adults with Down syndrome. Disability and Rehabilitation. 2016; 38(26):2575-83. [DOI:10.3109/0963 8288.2015.1137982] [PMID]

[20] Bressel E, Yonker JC, Kras J, Heath EM. Comparison of static and dynamic balance in female collegiate soccer, basketball, and gymnastics athletes. Journal of Athletic Training. 2007; 42(1):42-6. [PMID] [PMCID]

[21] Olejnik S, Algina J. Generalized eta and omega squared statistics: Measures of effect size for some common research designs. Psychological Methods. 2003; 8(4):434-47. [DOI:10.1037/1082-989X.8.4.434] [PMID]

[22] Shields N, Taylor NF, Dodd KJ. Effects of a communitybased progressive resistance training program on muscle performance and physical function in adults with Down syndrome: A randomized controlled trial. Archives of Physical Medicine and Rehabilitation. 2008; 89(7):1215-20. [DOI:10.1016/j.apmr.2007.11.056] [PMID]
[23] Jankowicz-Szymanska A, Mikolajczyk E, Wojtanowski W. The effect of physical training on static balance in young people with intellectual disability. Res Dev Disabil. 2012; 33(2):675-81. [DOI:10.1016/j.ridd.2011.11.015] [PMID]

[24] Carvalho RL, Almeida GL. Assessment of postural adjustments in persons with intellectual disability during balance on the seesaw. Journal of intellectual disability research : JIDR. 2009; 53(4):389-95. [DOI:10.1111/j.13652788.2008.01147.x] [PMID]

[25] Hale L, Bray A, Littmann A. Assessing the balance capabilities of people with profound intellectual disabilities who have experienced a fall. Journal of intellectual disability research : JIDR. 2007; 51(Pt 4):260-8. [DOI:10.1111/j.13652788.2006.00873.x] [PMID]

[26] Hill J, Leiszler M. Review and role of plyometrics and core rehabilitation in competitive sport. Curr Sports Med Rep. 2011;10(6):345-51. [DOI:10.1249/JSR.0b013e31823b3b94] [PMID]

[27] Kibler WB, Press J, Sciascia A. The role of core stability in athletic function. Sports medicine. 2006;36(3):189-98. [DOI:10.2165/00007256-200636030-00001] [PMID]

[28] Westendorp M, Hartman E, Houwen S, Smith J, Visscher C. The relationship between gross motor skills and academic achievement in children with learning disabilities. Res Dev Disabil. 2011;32(6):2773-9. [DOI:10.1016/j.ridd.2011.05.032] [PMID]

[29] Kibele A, Behm DG. Seven weeks of instability and traditional resistance training effects on strength, balance and functional performance. The Journal of Strength \& Conditioning Research. 2009;23(9):2443-50. [DOI:10.1519/ JSC.0b013e3181bf0489] [PMID]

[30] Calatayud J, Borreani S, Colado JC, Martín FF, Rogers ME, Behm DG, et al. Muscle activation during push-ups with different suspension training systems. Journal of Sports Science \& Medicine. 2014; 13(3):502. [PMID] [PMCID]

[31] Gaedtke A, Morat T. Effects of two 12-week strengthening programmes on functional mobility, strength and balance of older adults: Comparison between TRX suspension training versus an elastic band resistance training. Central European Journal of Sport Sciences and Medicine. 2016; 13(1):49-64. [DOI:10.18276/cej.2016.1-05]

[32] Gaedtke A, Morat T. TRX suspension training: A new functional training approach for older adults-development, training control and feasibility. International Journal of Exercise Science. 2015; 8(3):224-33. [PMID] [PMCID] 